\title{
Fish Farming Autonomous Calibration System
}

\author{
F. Lopes, H. Silva, J.M. Almeida, C. Pinho, E. Silva \\ INESC TEC \\ ISEP - School of Engineering, IPP, Porto, Portugal \\ email: fmlopes, hmsilva, jose.m.almeida, cpinho, eduardo.silva@inesctec.pt
}

\begin{abstract}
The fish farming industry is becoming widespread all over the world. By 2039 most of the fish we eat will come from the fish farming industry. In this work, we propose an autonomous robotic solution for indoor fish farming biomass estimation. Our proposed system moves silently on top of the tank borders using differential wheels and a structured light vision system (SLS). The SLS system is composed by a camera and two line lasers (projectors) equipped with a line beam that allows to obtain the fish depth profile present in the tank to perform biomass estimation. Results in laboratory and in real aquaculture environment with live fish are presented.
\end{abstract}

Keywords-Underwater vision system, Camera, Line laser, Structured light vision system, Fish Farming Calibration, Biomass estimation.

\section{INTRODUCTION}

The growth rate of world population $1.14 \%$ per year [1], making a total of 7.3 billion people in the world today and raising up to 10 billion in 2050 . This growth is putting lots of pressure in the renewal of the planet natural food resources.

The United Nations FAO organization [2] issues regulation that address fisheries management and development, by taking into account the knowledge and uncertainties about biotic and human components of marine ecosystems. The sole purpose of this approach is to plan, develop and manage fisheries in such a way that it can cope with human society needs, but also keep the full range of goods and services provided by marine ecosystems. Due to increasing demand for fish and fish proteins, fish farming has become a widespread activity all over the world. Already considered as a solid alternative to cover fish market demands, and according to a world bank report [3] it will be 2/3 of all world fish supplies by year 2030 .

We can roughly divide fish farming activities into two categories: extensive aquaculture or intensive aquaculture. While extensive aquaculture is usually related to outdoor fish farming, the intensive and semi-intensive aquaculture is more dedicated to indoor fish tank systems. In these kinds of fish growth systems, fish production per unit of surface is key, and companies try to maximize this key production item by mixing three fundamental elements: oxygen, fresh water and food. The way to do so, is by setting a very well structured fish tank production environment with controlled illumination, a continuous fresh water supply, and conducting periodical biological/chemical tests of all elements present in the production environment. They also conduct regular biomass and fish size evaluation procedures in order to check fish growth, a process denoted as biomass estimation.

The biomass estimation process is very labour intensive and increases the cost of production. On the other hand the density and biomass estimates are crucial for evaluating fish growth during its growth cycle. The statistical value is fundamental for fish farmers to estimate and adjust fish food dosage, medicine dosage, early detection of fish loss, but most importantly growth rates and food conversion factor appraisal to decide when is the best time to conduct financial transactions.

Nowadays, most of biomass estimation procedures in the fish farming industry are performed manually. Individual fish samples are collected from the indoor tanks to be measured and weighted. This procedure induces high fish mortality, illness, also causes fish stress affecting the fish biorhythm thus inducing a more slow growth rate.

In this paper we propose a novel robotic solution for indoor fish farming biomass estimation. Our solution is composed by two main physical components: First a robotic mechanical platform, that is placed on top of the borders of the underwater tank. The platform moves autonomously on top of the tank using differential wheels with encoder information. The information is then coupled with position lasers that measure platform travelled distance to a fixed target, usually placed at end of the tank; Second component is a mobile platform that moves on top of the mechanical platform and contains a structured light vision system (SLS), equipped with two lasers, a camera and a processing unit that performs 3D underwater mapping of the tank bottom using the triangulation principle [4], in an u-shape type movement.

This novel system was already tested in laboratory, and in a fish farming indoor production tank using live fish specimens. The obtained results allows us to conclude that our proposed solution is able to measure the biomass volume and the fish weight fare better than current solutions that are only based on manual samples collected by humans. The prototype is currently being upgraded to low its current weight and will start to work on a regular basis shortly.

The outline of this paper is the following, in section II a brief related work will be presented. Section III describes the robotic mechanical solution, the SLS system and its main components. Section IV contains tests results in laboratory environment and also in a real fish farm facility. Finally, in section $\mathrm{V}$ we discuss the conclusions and present some of our ideas to improve the system in future work.

\section{RELATED WORK}

The use of computer vision techniques for biomass estimation started in the mid 90s [5], [6], [7]. However, these systems still were equipped with low level of automation and required intense activity from operators. In early work, Foster et al. [5] used an underwater camera and image analysis tool to detect 
and count left over pellets. Petrell et al. [6] developed a system for estimating the mass of fish using stereo video cameras. In [7] the authors propose a video system for measuring the size and swimming speed in cages and tanks with a non-intrusive and offline system.

Most of the methods were applied to fix structures and only average biomass estimations were obtained. Furthermore at the time, there was no real time processing of the video images. Instead, video images were collected and its image content analysed in post-processing. As for instance, in [8] Lines et al. develop automatic techniques for identifying good images of fish in video frames and determine the outline of the fish in 3D space.Thereunto, they used a stereo vision system to extract linear dimensions of salmons and estimate their mass. Preliminary tests showed that the mean mass measurement error was $18 \%$ with a standard deviation of only $9 \%$. In other work, Costa et al. [9] tested a dual underwater camera system for counting and estimating fish length, while the fish was being transferred, the system reported length estimation calibration errors of less than $13 \%$ and biomass estimation error of roughly $50 \%$. The same authors, in [10] used a submersible dual camera module connected through two frame grabbers to a PC, the system used filtered and segmented images with a fixed threshold to obtain binary images. Image segments were analysed for area, major axis length and circularity. Afterwards, feature points in each stereo image pair are used to obtain their geometry. Fish-length estimation error, based on a single measurement of a model fish, was approximately $2 \%$.

In [11], [12] Martinez et al. develop a biomass estimation system using computer vision and robotic techniques, but the biomass estimation for indoor aquaculture was conducted over the water surface using pure visual based methods. With the increase in automation and machine vision systems, the combination of laser systems with visual methods for inspection tanks become widespread.

In [13] it was proposed a method to evaluate the spatial distribution of flat fish in raceway tanks using a laser and a digital camera. The aim of the system was to improve tank design and fish management. In [14] a laser scanning system is used to recover the biomass inspection of a fish tank. The SLS system, does not process the line laser images in real-time and the platform is not fully automatized, instead its manually pushed.

On the contrary, our system proposes a fully autonomous solution without human in the loop for obtaining 3D underwater mapping of an indoor Raceway tank system (RAS). With this solution and based on conservative estimates of an average fish density, is possible to obtain real-time estimates of the biomass in each tank. Moreover, the system can perform multiple scans and transform what is a painful manual process into a automatized simply process that can be executed several times per day, with much less measurement uncertainty.

\section{System ARChitecture}

Our system was designed to address the challenge of intensive biomass estimation in indoor RAS tanks. We developed a robotic platform that is mechanically adaptable to the RAS tanks. In Fig. 1, we can see our fish farming calibration system

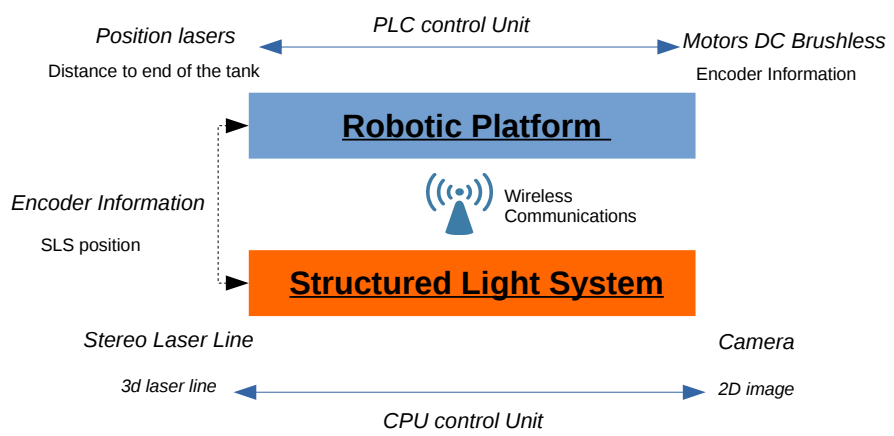

Fig. 1. Hardware Architecture Fish Farming Autonomous Calibration System

hardware architecture. It has two main components, the robotic platform (described in Section III-A) includes DC motors, sensors for providing odometry information and a processing unit. The structured light vision system (described in Section III-B) includes line lasers, camera and also a processing unit. The two components communicate between each by Wi-Fi network.

\section{A. Robotic Platform}

The robotic platform incorporates the following components:

- Omron PLC

- Mechanical Platform Position Lasers

- Brushless DC Motors with encoder information

- On-board Processing Unit

- $\quad$ LifePO4 batteries

- Wifi communications $2.4 \mathrm{GHz}$ module

For the remaining of the paper, we will use the tank reference frame: $\mathrm{XX}$ is to the right, $\mathrm{YY}$ is to front and $\mathrm{ZZ}$ is down.

In Fig. 2, an image of the robotic platform is displayed. The platform moves in the plane XY, parallel with the water plane. The platform movement in the $\mathrm{ZZ}$ axis is manual calibrated and its adjustable with the water column. The RAS tanks are very shallow depth ( between $15-25 \mathrm{~cm}$ ). The traction wheels are actuated using Brushless DC Motors that allow to perform movement in YY axis.

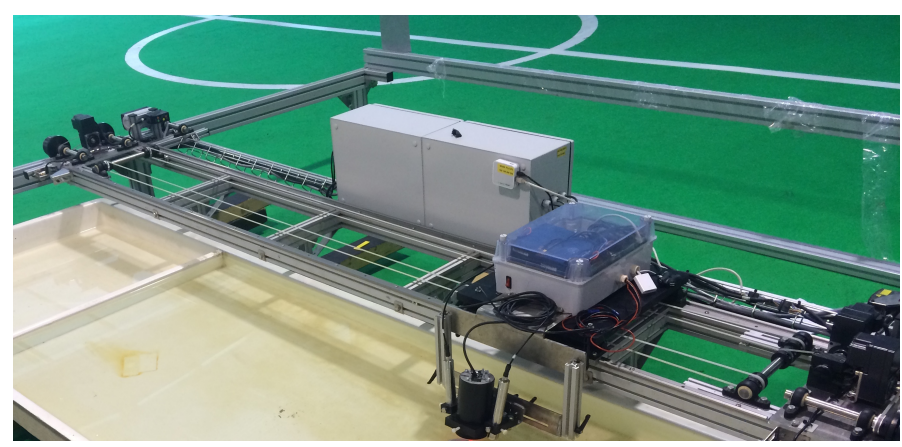

Fig. 2. Mechanical Platform. 


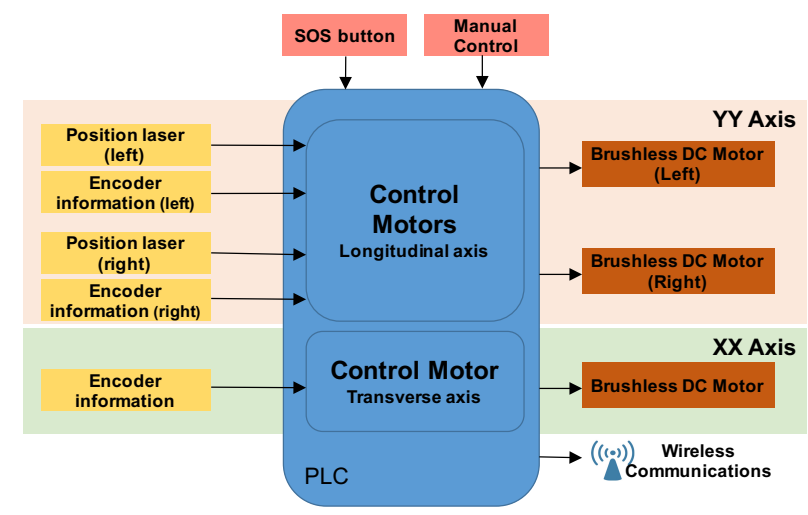

Fig. 3. Mechanical Platform Architecture.

The SLS is placed in a mobile frame that moves in XX axis, through a system with a DC Motor that operates with a "timing belt" mechanism. The SLS contains a processing unit synchronized with the PLC that is controlling the robotic platform, and communicates using the Wi-Fi network.

In order to obtain the global platform position in the tank reference frame, position lasers were installed in the robotic platform and artificial targets are placed at end of tank. This sensing mechanism allows us to keep track of the total travel distance of the robotic platform in the tank, the lasers are placed on the sides of the robotic platform. The position lasers allow traction control in $\mathrm{YY}$ axis and avoid other situations such as slipping wheel. In this situation the system tries to compensate the motion or stops the scan. The PLC is responsible for receiving lasers and encoders information, control the platform movement in XX and YY axis and send odometry information to the SLS processing unit.

The robotic platform operation in automatic mode can be described by: The robotic platform is placed in a initial reference point with the SLS on the side of the tank. A scan is performed, by moving the SLS in XX axis until it reaches the other side of the tank. The SLS continuously captures and process the images while receiving odometry information from the PLC. Then, a movement is performed in the YY axis with a predetermined step, which is constant through out the length of the tank. Followed by a scan is while the SLS is moving in the opposite direction. This procedure, based on a u-shape movement is repeated until it scans the entire tank.

The robotic platform is mechanically built using aluminium and stainless steel to prevent corrosion caused by the hostile environment on fish farming industries. As for the SLS unit its built using waterproof housings.

\section{B. Structured Light Vision System}

The mobile structured light vision system (SLS), contains the following components:

- $\quad$ NUC Intel i5 $2.70 \mathrm{GHz}$ processing unit

- $\quad$ IDS UI-3240C $1.3 \mathrm{Mp}$

- 2 Global Lyte Mv Lasers $635 \mathrm{~nm}$ wavelength (red) $5 \mathrm{~mW}$ power with waterproof Housing

- $\quad$ LifePO4 batteries
- Wifi communications $2.4 \mathrm{GHz}$ module

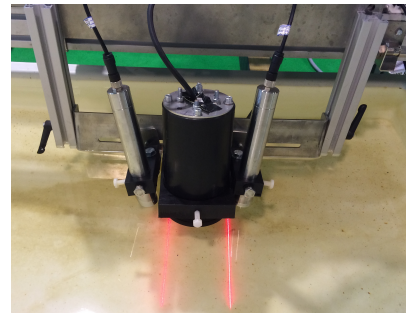

(a) Structured Light Vision System Camera and red lasers.

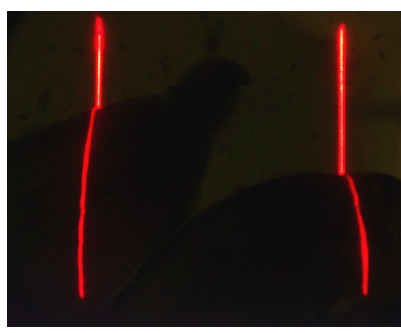

(b) Snapshot Structured Light Vision Image, red laser line deformation in the presence of a fish.
Fig. 4. Structured Light Vision System.

The SLS unit consists of: one camera and two red line lasers, both with waterproof housings, see in Fig. 4(a). The processing unit is responsible for acquiring and processing the 2D images, receive odometry information and compute the triangulation for obtaining all points of the line laser in 3D reference coordinates.

\section{Calibration}

One of the critical issues that needed to be solved in order for the SLS system to work, was the calibration between the laser projectors and the camera. In [15] we proposed two SLS calibration methods, one based on the cross-ratio principle, while the other was based on the robust estimation of the laser line projection in the camera reference frame. The methods were tested both in dry and underwater environments and the line projection method achieved $2 \mathrm{x}$ times smaller errors than the cross-ratio method. The results obtained show that error for the line projection method is about $1 \mathrm{~mm}$, see [15] results section.

\section{3D Modelling}

The SLS allows us to obtain 3D information from the flats fish present in an rectangular indoor tank. In Fig.5 we can see the SLS software architecture. The main purpose is to obtain synchronized time stamp images with dual line laser projection information. Flatfish species are not prone to sudden movements, the fish usually stays in the bottom of the tank in layers. Therefore, with a SLS constantly submerged underwater performing u-shaped laser scans is possible to capture the fish profile layer in the tank.

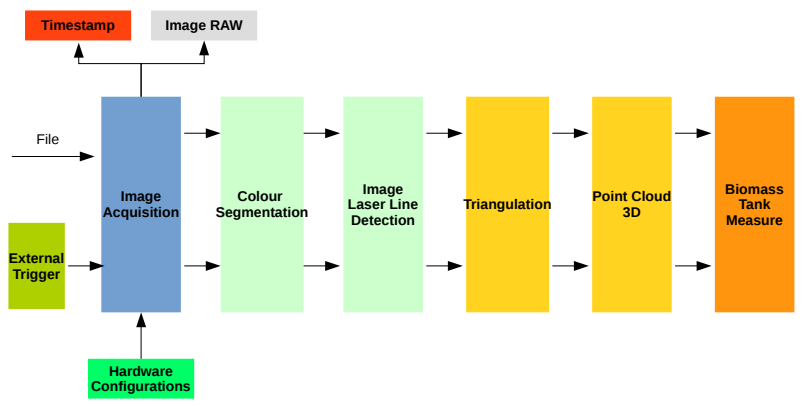

Fig. 5. SLS Software Architecture 


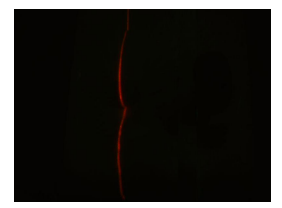

(a) Original image.

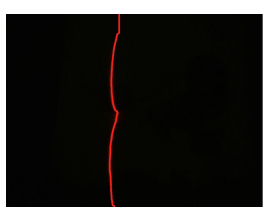

(b) Image with all pixels (c) Snapshot of some with red component. image lines - Gaussian Kernel result.
Fig. 6. SLS - Line laser detection.

Prior to be able to use the SLS for obtaining accurate 3D underwater measurement, there is the need to perform a global system calibration. So, in this step we need to load the camera intrinsic parameters and the plane of each projector position in camera reference frame, obtained in calibration step. Initially, it may be necessary to define configuration parameters such as: frame rate, exposure time or a threshold value for the color segmentation.

The method which allows to obtain the 3D point cloud is the following. First, we start image acquisition procedure followed by an image segmentation method where only the red component is preserved. The next step concerns the detection of line lasers in the 2D image, as shown in Fig. 6(b). Using a Gaussian Kernel is possible to obtain the pixel with greater color intensity for each horizontal line of the image, as shown in Fig. 6(c).

After obtaining the line laser in the 2D image frame and previously knowing the camera/laser calibration parameters, is possible to obtain 3D point information, using the triangulation principle between the camera viewpoint and the light projector. For more detail please see our previous work [16]. For computing the 3D point cloud in the tank reference frame is necessary to use odometry information. The profile layer gives us fish height in the tank reference frame, compared to a previously known measure of the profile tank height only with water. Having determine this profile we can then estimate the biomass volume of the fish present in the tank.

\section{RESUlTS}

\section{A. Laboratory Experiment}

For testing our fish farming autonomous calibration system we started by performing some laboratory experiments. A fish tank measuring $3 \times 1 \mathrm{~m}$ with depth variations between $12 \mathrm{~cm}$ and $15 \mathrm{~cm}$, was used in the experiments. In Fig.7 is possible to see an image of the experimental setup.

For this experiment we used two different types of fish: flounder and turbot. The working principle consisted on first do the 3D underwater mapping of the tank only with water. Then we placed dead fish and perform the 3D mapping of the fish using the SLS. Based on the comparison between both measures is possible to extract the fish biomass volume contained in the tank.

In the first test, a sample with 4 flounder with $0,956 \mathrm{~kg}$ of weight was used. In Fig.8, is possible to see the fish sample, as well as, the corresponding 3D point cloud result.
Our system measures the overall biomass volume present in a given tank. However a potential client/producer, is actually interested in knowing the overall fish biomass weight (M) present in the tank. Therefore, is necessary to relate the actual fish weight with his density. For doing so, we collected a fish sample, and use a container with known dimensions and known volume of the water column. We placed all the fish samples inside of the container in order to measure the difference of water column height to calculate the density of the fish.

Afterwards, the weight of this sample is obtained using equation (1), where $\mathrm{M}$ represent the biomass weight, $\mathrm{V}$ the biomass volume, measured by the system and $\rho$ the biomass density.

$$
\rho=\frac{M}{V}
$$

The results presented in Table I, show that our approach has about $5 \%$ of relative error in estimate the biomass weight.

TABLE I. COMPARISON OF THE TOTAL BIOMASS WEIGHT MEASUREMENTS BETWEEN THE MANUAL CALIBRATION AND THE SCAN SYSTEM IN INESC TEC LABORATORY - FLOUNDER

\begin{tabular}{|c|c|}
\hline Number of fish & 4 \\
\hline Biomass density $\left(\mathbf{k g} / \mathbf{m}^{\mathbf{3}}\right)$ & 1020 \\
\hline
\end{tabular}

\begin{tabular}{|c|c|c|c|}
\cline { 2 - 4 } \multicolumn{1}{c|}{} & Weight (kg) & $\begin{array}{l}\text { Absolute } \\
\text { Error }(\mathbf{k g})\end{array}$ & $\begin{array}{l}\text { Relative Er- } \\
\text { ror (\%) }\end{array}$ \\
\hline Manual Calibration & 0,956 & - & - \\
\hline SCAN & 0,907 & 0,049 & 5,125 \\
\hline
\end{tabular}

Analogously, the same tests were performed for other type of flat fish, the turbot. For this test, we use a sample of 5 turbot weighing about $4,850 \mathrm{~kg}$. In Fig. 9 is shown the fish sample used and the corresponding 3D point cloud result. The results presented in Table II, show that our approach has about $8 \%$ of relative error in estimating the biomass weight.

In Fig. 10 a result of 3D scan of the fish turbot is presented. In this image is possible to see that the tank was about 125 $\mathrm{mm}$ and the fish maximum thickness is approximated $40 \mathrm{~mm}$.

\section{B. Indoor Fish Farm Experiment}

The fish farming calibration system was experimented in a live indoor aquaculture farming industry. For this purpose a

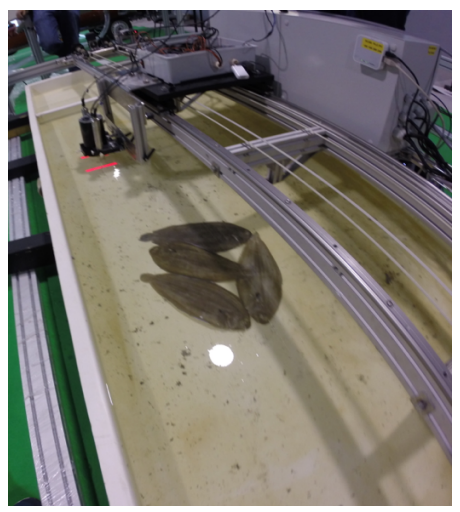

Fig. 7. Experimental Setup Laboratory Tank 

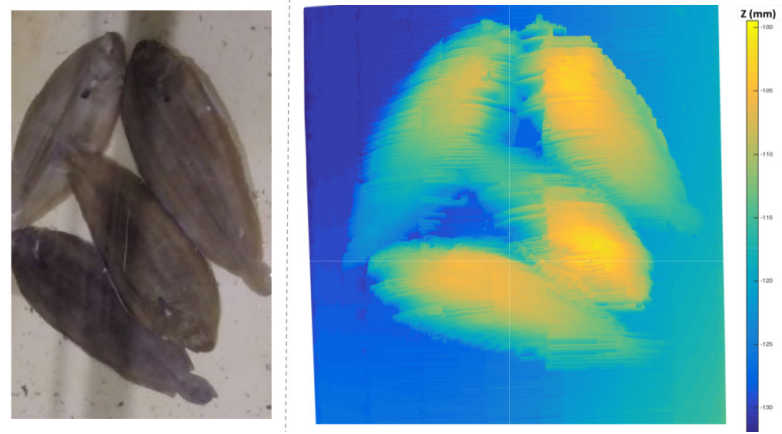

Fig. 8. Point cloud 3D scan result of the fish farming calibration in laboratory tank
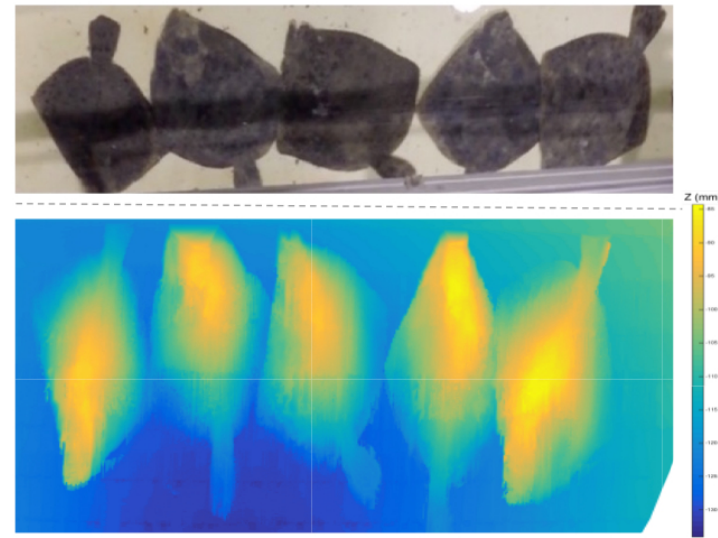

Fig. 9. Point cloud 3D scan result of the fish farming in laboratory tank.

TABLE II. COMPARISON OF THE TOTAL BIOMASS WEIGHT MEASUREMENTS BETWEEN THE MANUAL CALIBRATION AND THE SCAN SYSTEM IN INESC TEC LABORATORY - TURBOT

\begin{tabular}{|c|c|c|c|}
\hline \multirow{3}{*}{\begin{tabular}{|c|} 
Number of fish \\
Biomass density $\left(\mathrm{kg} / \mathrm{m}^{3}\right)$ \\
\end{tabular}} & 5 & & \\
\hline & 1200 & & \\
\hline & Weight (kg) & $\begin{array}{l}\text { Absolute } \\
\text { Error (kg) }\end{array}$ & $\begin{array}{l}\text { Relative Er- } \\
\text { ror }(\%)\end{array}$ \\
\hline Manual Calibration & 4,850 & & - \\
\hline SCAN & 4,470 & 0,380 & 7,833 \\
\hline
\end{tabular}

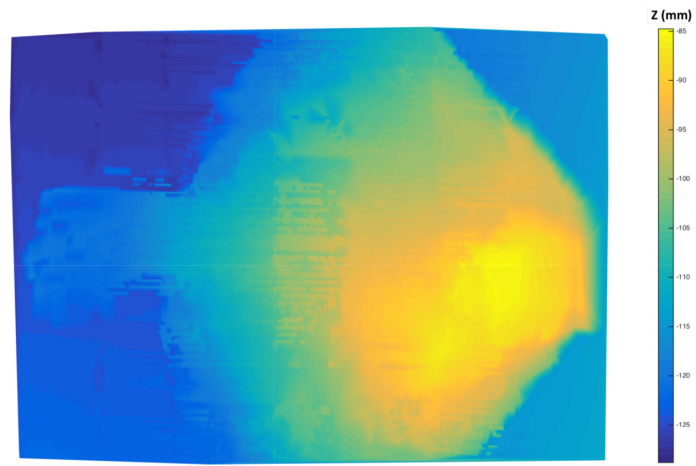

Fig. 10. Point cloud 3D scan result of the fish turbot.

section of a large $40 \times 3.5 \mathrm{~m}$ tank, with $2.80 \times 3.50 \mathrm{~m}$ was selected. In Fig. 11 we can see the system operating in a RAS production tank.

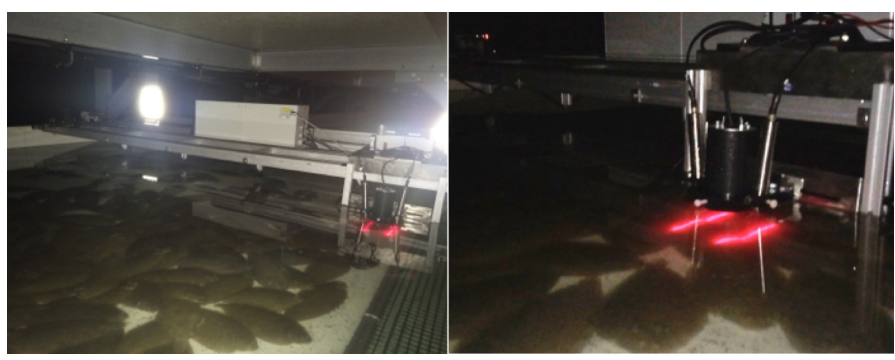

Fig. 11. Experimental Setup Indoor Tank.

The amount of biomass available in the tank was manually calibrated for ground-truth estimation purposes. In this test, performed in an actual aquaculture environment, we used 209 flounders with $69,700 \mathrm{~kg}$ of total weight. Having such a large quantity of fish it was impossible to know the real mean value of its biomass density.

Therefore, the biomass weight was estimated using density values, between $1200 \mathrm{~kg} / \mathrm{m}^{3}$ and $1300 \mathrm{~kg} / \mathrm{m}^{3}$. Even not being to thorough, since these are indicative values for live fish individuals, it allows us to have an approximated idea of the measurement errors associated, see Table III. The results show that our approach has between $10 \%$ and $17 \%$ of relative error in biomass volume in real aquaculture environment. These results will have to be further validated in future work, taking into consideration the amount of fish present in a given tank and their growth during their life cycle. Also in the future, the mean value for biomass density will be estimated using statistical databases. The database will be generated by multiples scans of the same individuals in the same tank for estimate an approximate real biomass density value, and also by weighing all the fish in the tank priory to their sale.

In Fig. 12 is displayed the final result of the 3D point cloud of the fish farming indoor tank experiment.

TABLE III. COMPARISON OF THE TOTAL BIOMASS WEIGHT MEASUREMENTS BETWEEN THE MANUAL CALIBRATION AND THE SCAN SYSTEM IN THE FISH FARMING FACILITY

\begin{tabular}{|c|c|c|c|}
\hline Number of fish & 209 & \multirow[b]{3}{*}{$\begin{array}{l}\text { Absolute } \\
\text { Error (kg) }\end{array}$} & \multirow[b]{3}{*}{$\begin{array}{l}\text { Relative Er- } \\
\text { ror }(\%)\end{array}$} \\
\hline \multirow[t]{2}{*}{ Biomass density $\left(\mathrm{kg} / \mathrm{m}^{3}\right)$} & 1200 & & \\
\hline & Weight (kg) & & \\
\hline Manual Calibration & 69,700 & - & - \\
\hline SCAN & 57,749 & 11,950 & 17,146 \\
\hline \multirow[t]{2}{*}{ Biomass density $\left(\mathrm{kg} / \mathrm{m}^{3}\right)$} & 1300 & & \\
\hline & Weight (kg) & $\begin{array}{l}\text { Absolute } \\
\text { Error (kg) }\end{array}$ & $\begin{array}{l}\text { Relative Er- } \\
\text { ror }(\%)\end{array}$ \\
\hline Manual Calibration & 69,700 & - & - \\
\hline SCAN & 62,562 & 7,138 & 10,241 \\
\hline
\end{tabular}

\section{CONCLUSION}

The aquaculture industry is turning rapidly into a billion dollar industry. Therefore is important to maintain standards that allow fish to grow in a sustainable manner. Currently there are lots of interests in optimizing the growth with parameters like food, water quality, temperature and oxigen in the tank. 


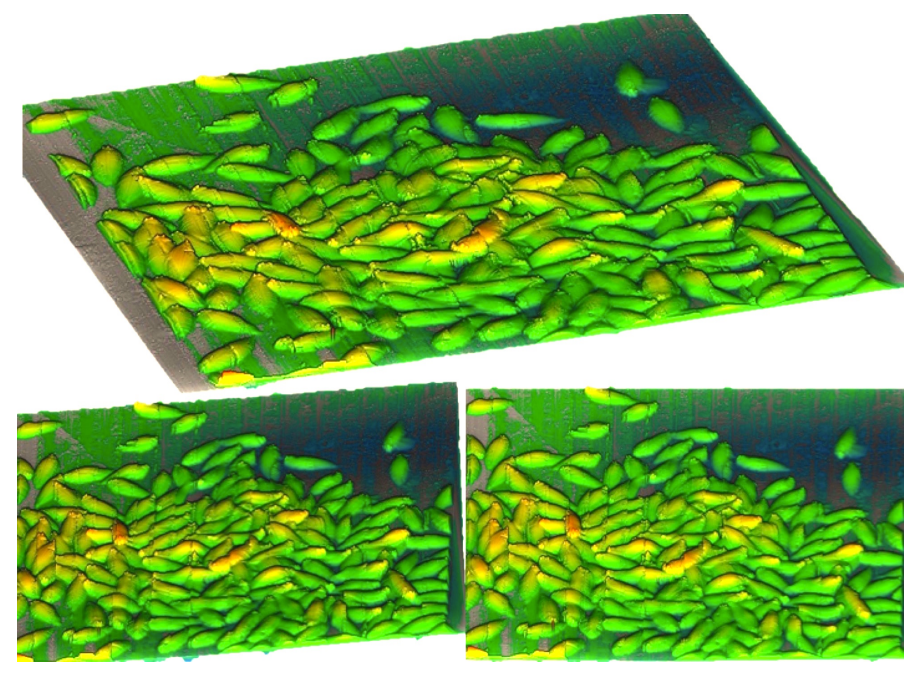

Fig. 12. PointCloud 3D scan result of the fish farming indoor tank experiment

The biomass estimation procedure is usually performed manually, originating fish mortality, illness, and causing fish stress that induces a slow growth.

In this work we have presented an autonomous system for indoor fish farming biomass estimation. Our robotic mechanical platform that is placed and moves on the borders of a RAS tank contains a moving platform with our custom designed structured light vision system. The 3D underwater laser scanning technique is a promising tool for estimating the biomass weight, and is an alternative to the common manual measured system.

For proof concept our system was tested in laboratory experiments, and in these tests we use two different types of fish: flounder and turbot. In laboratory experiments, the results show that our approach has a maximum $8 \%$ of relative error in estimating the biomass weight. The fish farming calibration system was also experimented in a live indoor aquaculture farming industry and the results show that our approach has about $15 \%$ error in overall biomass weight in real aquaculture environment.

In future work, there is need to integrate an inertial measurement unit in the SLS system to reduce the position and attitude errors of the robotic mechanic platform. One of the error sources is for not taking into consideration the robotic platform attitude in the tank. Also mechanical redesign towards a weight reduction of the all system is currently under way, to allow an easy assembly and mobility inside the indoor fish farm.

\section{ACKNOWLEDGMENT}

"This work is financed by CORDIS - Community Research and Development Information Service through H2020EU.4.b. - Twinning of research institutions within Project Strongmar ID: 692427 and by National Funds through the FCT - Fundação para a Ciência e a Tecnologia (Portuguese Foundation for Science and Technology) as part of project UID/EEA/50014/2013."

\section{REFERENCES}

[1] http://www.worldometers.info/world-population/

[2] http://www.fao.org/docrep/005/Y4470E/Y4470E00.HTM

[3] http://documents.worldbank.org/curated/en/2013/12/18882045/fish2030-prospects-fisheries-aquaculture

[4] Hartley, R. I. and Zisserman, A.," Multiple View Geometry in Computer Vision", Second Edition, 2004,Cambridge University Press, ISBN: 0521540518

[5] M. Foster, R. Petrell, M.R. Ito, R. Ward, "Detection and counting of uneaten food pellets in a sea cage using image analysis", Aquacultural Engineering, Volume 14, Issue 3, Pages 251-269, ISSN 0144-8609, (1995).

[6] R.J. Petrell, X. Shi, R.K. Ward, A. Naiberg, C.R. Savage, ”Determining fish size and swimming speed in cages and tanks using simple video techniques", Aquacultural Engineering, Volume 16, Issues 1-2, Pages 63-84, ISSN 0144-8609, ( March 1997).

[7] K.P. Ang, R.J. Petrell, "Control of feed dispensation in seacages using underwater video monitoring: effects on growth and food conversion". In: Aquacultural Engineering 16, pp.45-62, (1997).

[8] J.A. Lines, R.D. Tillett, L.G. Ross, D.Chan, S. Hockaday, N.J.B McFarlane, "An automatic image-based system for estimating the mass of freeswimming fish". In: Computers and Electronics in Agriculture, pp.151168, (April 2001)

[9] C. Costa, M. Scardi, V. Vitalini S. Cataudella, "A dual camera system for counting and sizing Northern Bluefin Tuna (Thunnus thynnus; Linnaeus, 1758) stock, during transfer to aquaculture cages, with a semi automatic Artificial Neural Network tool”. In: Aquaculture 291, pp.161-167, (2009).

[10] C. Costa, A. Loy, S. Cataudella, D. Davis, M. Scardi, "Extracting fish size using dual underwater cameras". In: Aquaculture Engineering 35, pp.218-227, (2006).

[11] C. Serna , A. Ollero, "A stereo vision system for the estimation of biomass in fish farms". Proceedings of the 6th IFAC Symposium, pp.185191. Berlin, Germany, 8,9 October, (2001).

[12] J. R. Martinez-de Dios, C. Serna, A. Ollero. "Computer vision and robotics techniques in fish farms". Robotica 21, 233-243, (June 2003).

[13] J. Oca, S. Duarte, L. Reig. "Evaluation of spatial distribution of flatfish by laser scanning", Aquaculture Europe 2007. Istanbul, Turkey: European Aquaculture Society, 24-27 p. 157, (2007).

[14] C. Almansa, L. Reig, J. Oca, "Use of laser scanning to evaluate turbot (Scophthalmus maximus) distribution in raceways with different water velocities, Aquacultural Engineering, Volume 51, Pages 7-14, ISSN 0144-8609, (2012).

[15] F. Lopes, H. Silva, J. Almeida, A. Martins ,E. Silva, "Structured Light System Calibration for Perception in Underwater Tanks", IbPRIA 2015: 7th Iberian Conference on Pattern Recognition and Image Analysis Santiago de Compostela, Spain. June 17-19 (2015)

[16] F. Lopes, H. Silva, J. Almeida, E. Silva, "Structured Light System for Underwater Inspection Operations", OCEANS'15 MTS/IEEE Genova, Italy, 18-21 May (2015) 